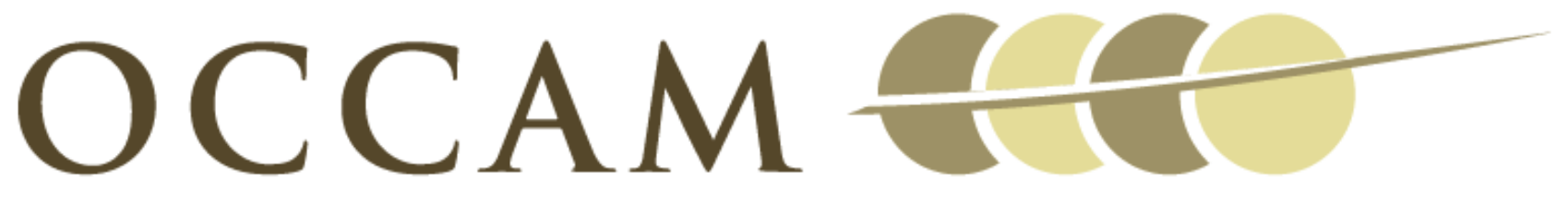

OXFORD CENTRE FOR COLLABORATIVE APPLIED MATHEMATICS

Report Number 10/36

Sequential Inverse Problems Bayesian Principles and the Logistic Map Example

by

Lian Duan, Chris L. Farmer, and Irene M. Moroz

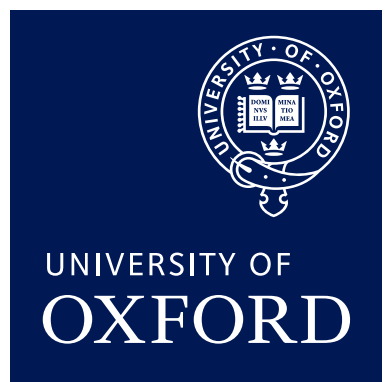

Oxford Centre for Collaborative Applied Mathematics Mathematical Institute 24 - 29 St Giles'

Oxford

OX1 3LB

England 



\title{
Sequential Inverse Problems Bayesian Principles and the Logistic Map Example
}

\author{
Lian Duan*, Chris L. Farmer* and Irene M. Moroz \\ * Oxford Centre for Collaborative Applied Mathematics, Mathematical Institute, University of Oxford, OX1 3LB \\ ${ }_{\dagger}$ Oxford Centre for Industrial and Applied Mathematics, Mathematical Institute, University of Oxford, OXI 3LB
}

\begin{abstract}
Bayesian statistics provides a general framework for solving inverse problems, but is not without interpretation and implementation problems. This paper discusses difficulties arising from the fact that forward models are always in error to some extent. Using a simple example based on the one-dimensional logistic map, we argue that, when implementation problems are minimal, the Bayesian framework is quite adequate. In this paper the Bayesian Filter is shown to be able to recover excellent state estimates in the perfect model scenario (PMS) and to distinguish the PMS from the imperfect model scenario (IMS). Through a quantitative comparison of the way in which the observations are assimilated in both the PMS and the IMS scenarios, we suggest that one can, sometimes, measure the degree of imperfection.
\end{abstract}

Keywords: Logistic Map, Bayesian Filter, Grid Based Method, Hellinger Distance, Distinguishing the Prefect and Imprefect Scenarios PACS: $02.50 . \mathrm{Ng}, 05.45 . \mathrm{Tp}, 02.50 . \mathrm{Cw}, 02.50 . \mathrm{Le}, 05.45 . \mathrm{Ac}$

\section{INTRODUCTION}

In many problems such as that of weather or hydrocarbon reservoir forecasting one must characterise the initial state of knowledge using a probability density function (pdf). The non-linearity of the models makes it difficult to evolve this initial pdf and makes it difficult to assimilate any new measurements. The logistic map: $x_{t+1}=1-a x_{t}^{2}$, with $x_{t} \in[-1,1], a \in[0,2]$ provides a very simple example of this situation. The inverse problem requires an estimation of $x_{t}$ and $a$, given a sequence of noisy observations, $s_{t}=x_{t}+v_{t}$, where $v_{t}$ is the noise. Methods for solving inverse problems associated with such chaotic or unstable systems starts with Berliner [1], who introduces the inverse problem for the logistic map and proposes a Bayesian solution. The maximum a posteriori method is used and gives good results when the data are observed from the true system, a situation termed the perfect model scenario (PMS) by Judd and Smith [2]. The more realistic case, of course, is when observations are taken from an unknown physical system, which differs from the model. We are then in the imperfect model scenario (IMS) of Judd and Smith [3].

In this paper, we derive the exact analytical solution of the Bayesian filter for the logistic map. Using numerical experiments in the PMS, the performance of the Bayesian filter is examined with a grid-based method. Finally, we use the Hellinger distance [6] between the prior and posterior pdfs, to distinguish between the PMS and IMS scenarios.

\section{EXACT SOLUTION OF BAYESIAN FILTER}

Assume the logistic map, $x_{t+1}=1-a x_{t}^{2}$, with $t \in\{0,1,2 \ldots\}, x_{t} \in[-1,1]$ and $a \in[0,2]$ has been chosen to model a time series of noisy observations $s_{t}$, given by $s_{t}=x_{t}+v_{t}$, where the noise $v_{t}$ is sampled independently from a Gaussian distribution, $\pi_{\sigma}$, with zero mean and known variance $\sigma^{2}$. The vector of observations $\left\{s_{m}\right\}_{m=1}^{M}$ is denoted by $S_{M}$. Note the choice not to observe the initial state. Finally, we assume that a prior joint pdf, $\pi_{0}\left(x_{0}, a\right)$, for $x_{0}$ and $a$ has been provided. We will focus on the following two problems:

\section{The prediction problem}

Suppose that a pdf $\pi\left(x_{t}, a \mid S_{t}\right)$ has been found at time $t$. Using independence of the variables and properties of the delta function, the prior pdf, $\pi\left(x_{t+1}, a \mid S_{t}\right)$, at time $t+1$ is given by

$$
\pi\left(x_{t+1}, a \mid S_{t}\right)=\int_{-1}^{1} \pi\left(x_{t+1}, x_{t}, a \mid S_{t}\right) d x_{t}=\int_{-1}^{1} \pi\left(x_{t+1} \mid x_{t}, a\right) \pi\left(x_{t}, a \mid S_{t}\right) d x_{t} .
$$


Substituting for $x_{t}$ from the logistic map into equation (1) and evaluating the integral using properties of the delta function, we obtain

$$
\tilde{\pi}_{t+1}\left(x_{t+1}, a \mid S_{t}\right)=\frac{1}{\sqrt{4 a\left(1-x_{t+1}\right)}}\left\{\pi_{t}\left(\sqrt{\frac{1-x_{t+1}}{a}}, a \mid S_{t}\right)+\pi_{t}\left(-\sqrt{\frac{1-x_{t+1}}{a}}, a \mid S_{t}\right)\right\},
$$

where $\tilde{\pi}_{t+1}=0$ for $x_{t+1}<(1-a)$ and $t>0$.

\section{The filtering problem}

Suppose that a measurement $s_{t+1}$ is received at time $t+1$. Standard methods from probability theory give

$$
\pi_{t+1}\left(x_{t+1}, a, s_{t+1} \mid S_{t}\right)=\pi_{t+1}\left(s_{t+1} \mid x_{t+1}, a, S_{t}\right) \tilde{\pi}_{t+1}\left(x_{t+1}, a \mid S_{t}\right)=\pi_{t+1}\left(s_{t+1} \mid x_{t+1}, a\right) \tilde{\pi}_{t+1}\left(x_{t+1}, a \mid S_{t}\right) .
$$

Substituting for the observation model and equation (2) into equation (3), we obtain

$$
\pi_{t+1}\left(x_{t+1}, a, s_{t+1} \mid S_{t}\right)=\frac{\pi_{\sigma}\left(x_{t+1}-s_{t+1}\right)}{\sqrt{4 a\left(1-x_{t+1}\right)}}\left\{\pi_{t}\left(\sqrt{\frac{1-x_{t+1}}{a}}, a \mid S_{t}\right)+\pi_{t}\left(-\sqrt{\frac{1-x_{t+1}}{a}}, a \mid S_{t}\right)\right\},
$$

where $\pi_{t+1}=0$ for $x_{t+1}<(1-a)$ and $t>0$. Equation (4) is similar in form to that for the Perron-Frobenius map [4]. Finally, applying Bayes' rule, one deduces that

$$
\pi_{t+1}\left(x_{t+1}, a \mid S_{t+1}\right)=\frac{\pi_{\sigma}\left(x_{t+1}-s_{t+1}\right) \tilde{\pi}_{t+1}\left(x_{t+1}, a \mid S_{t}\right)}{\int_{0}^{2} \int_{-1}^{1} \pi_{t+1}\left(x_{t+1}, a \mid S_{t+1}\right) d x_{t+1} d a}
$$

\section{NUMERICAL EXPERIMENTS IN THE PERFECT MODEL SCENARIO}

The grid based method (GBM) proceeds by constructing a uniform $n_{x}$ by $n_{a}$ grid of cell $(i, j)$ where $i \in\left[1, n_{x}\right]$ and $j \in\left[1, n_{a}\right]$ on the region $[-1,1] \times[0,2]$. Associated with each cell is a constant value of the initial prior density, giving rise to a piecewise constant approximation to the initial prior in each cell. At time $t$ we assume that a piecewise constant approximation is available. This is then updated at time $t+1$ at the grid points, with equation (4), using the actual values of the observations. After all the points are evaluated, the result is normalized by dividing by the integral with respect to $x$ and $a$. When in predictive mode equation (2) is used.

We use the choice in [5] of $a=1.85, x_{0}=0.3$ and $\sigma=0.1$ in the numerical GBM experiments. We take uniform priors for $a$ and for $x$ with $x \in[-1,1]$ and $a \in[0,2]$. The experiment was run for 100 time steps on a $1000 \times 1000$ grid, with assimilation of observations at the end of each of the first 50 time steps. As already noted, we did not assimilate the observation of the initial state. As data was assimilated, the pdfs both before and after assimilation were analyzed. For illustrative purposes, the expectation value before assimilation was calculated, together with the 0.05 and 0.95 quantiles. After 50 time steps the experiment switched to prediction only, and data assimilation ceased.

Fig. 1 shows the observations (plotted with plus signs) and the underlying true state, without observational noise, (plotted as circles). As an estimate of the true system state, its expectation value (after assimilating the measurement) is used and plotted as crosses. The range of values of $x_{t}$, bounded by the 0.05 and 0.95 quantiles, is also plotted as gray blocks. The results are shown for the first 60 time steps. To start with, the Bayesian filter is learning the pdf for the model, here the PMS. After a few time steps the results are quite good. When assimilation is halted, at first the predictions remain good, but we see that the quantiles rapidly depart from one another, before spanning the full range of possible values fori $x_{t}$. This is in accord with our intuitions regarding chaotic systems.

Fig. 2 shows evolution of the pdf $\pi_{t}\left(a \mid S_{t}\right)$ at intervals of 10 time steps. We remark that $\pi_{t}\left(a \mid S_{t}\right)$ stays unchanged when assimilation is halted, which can also be proved using equation (2). The exact value for $a$ is 1.85 . The expectation (or average) value of $a$ after assimilation of the measurement at time step 50, is 1.859 with the 0.05 quantile at 1.848 and the 0.95 quantile at 1.888 . The true value for $x_{0}$ is 0.009946 . Its 0.05 quantile is at -0.029 and its 0.95 quantile is at 0.276 . It therefore appears that Bayesian statistics recovers both the initial state and the parameter value quite well in the PMS, even for a high level of observational noise. 


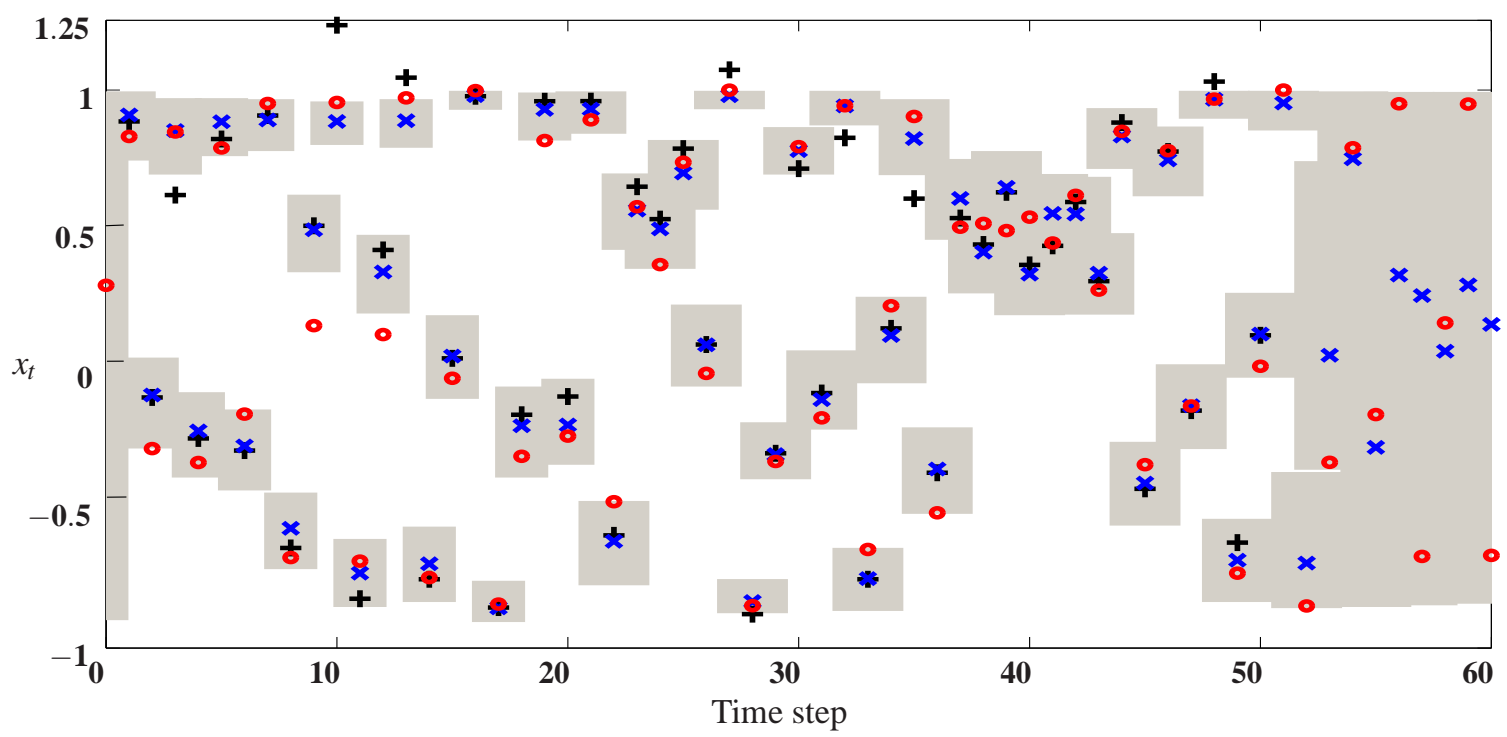

FIGURE 1. Plots of the expectation of $x_{t}$ (crosses) with $\sigma=0.1$ to $t=60$, the exact states of $x_{t}$ (circles), the observations $s_{t}$ (pluses) in the PMS and 0.05 and 0.95 quantiles (gray).

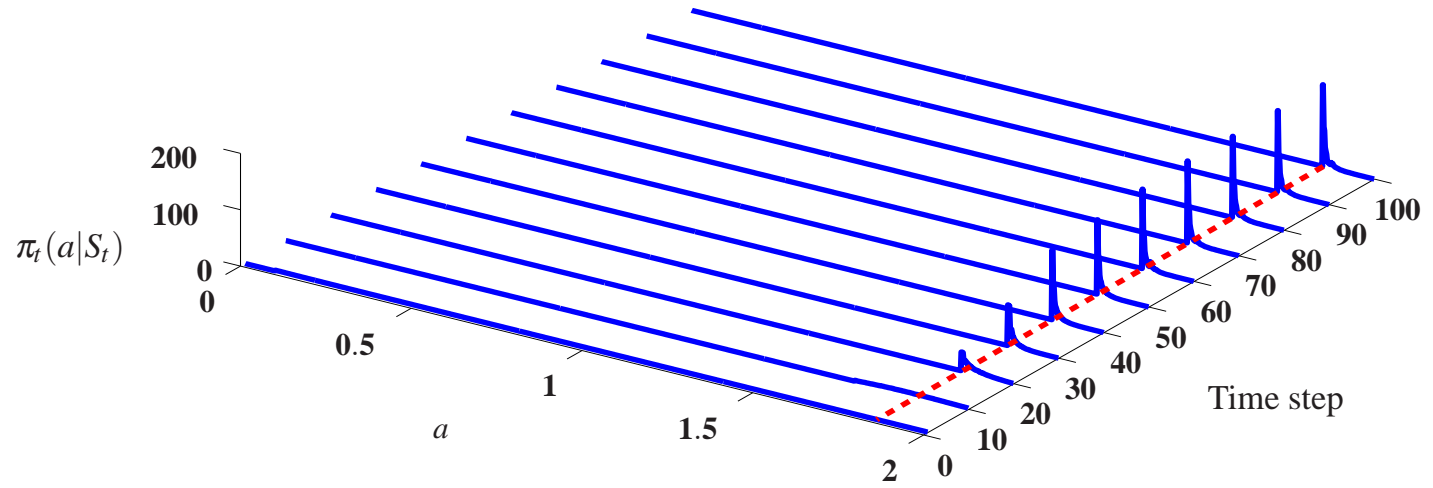

FIGURE 2. Predictive pdf for $a$ in the high noise PMS.

\section{DISTINGUISHING THE IMPERFECT FROM THE PERFECT MODEL SCENARIO}

In the IMS, the logistic map will again be used as the postulated model. The true system is taken to be a constant state, with observations subject to noise. The true state is chosen to be a value not coinciding with an attractor of our logistic map. We take $x_{n}=0.3$.

We need a test, designed to assess in which scenario we find ourselves. The suggestion proposed here is to evaluate the 'distance' between the predictive pdf and the pdf after assimilation at each time step during the assimilation phase. In this paper we use the Hellinger distance [6], defined by the integral:

$$
h_{t}=\frac{1}{2} \int_{-1}^{1} \int_{0}^{2}\left(\sqrt{\tilde{\pi}_{t}}-\sqrt{\pi_{t}}\right)^{2} d x_{t} d a_{t} .
$$




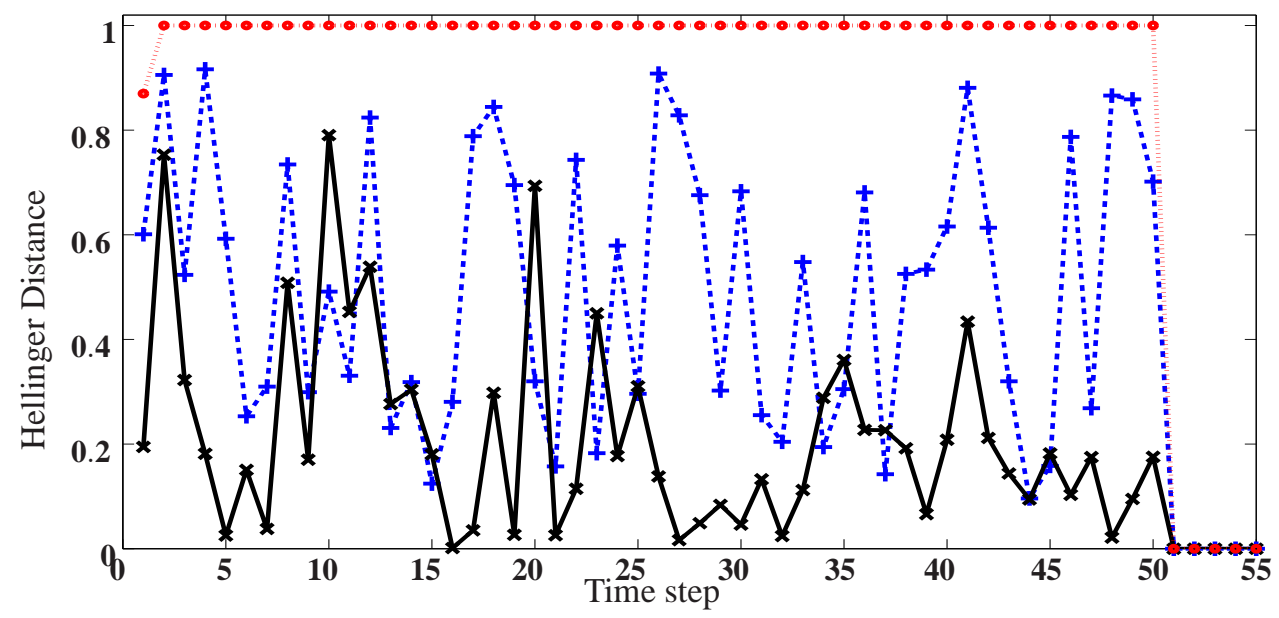

FIGURE 3. Hellinger distance distinguishing the IMS from the PMS. The high noise $(\sigma=0.1)$ PMS is shown as crosses, the high noise IMS $(\sigma=0.1)$ is shown as pluses and the low noise $(\sigma=0.01)$ IMS is shown as circles.

Fig. 3 shows $h_{t}$ as a function of time for the PMS, as indicated by crosses, and the IMS with both a high $(\sigma=0.1)$ noise, as indicated by the pluses, and a low ( $\sigma=0.01)$ noise (indicated by circles). The Hellinger distance is generally small for the PMS, showing that the data does not update the retrieval beyond correcting for error growth due to chaos. However, in the IMS low noise case $(\sigma=0.01)$, the Hellinger distance is nearly always close to unity; there is therefore no useful information in the data relative to the chosen model. In other words, the model (here, the logistic map) has no predictive capacity whatsoever, and so is inadequate. It should be noted, however, that in other applications a small Hellinger distance could be an indication of irrelevant data rather than a good or perfect model. success for the model.

\section{DISCUSSION}

In this paper we derived an exact expression for the Bayesian filter applied to the logistic map. Using numerical integration accurate estimates of both $x_{0}$ and $a$ were possible in the PMS. By including a method of model criticism, it has been demonstrated that the analysis can indicate in which scenario - perfect or imperfect - we are located. The conclusion must be that people with good priors and good models will make better predictions than those without such knowledge. This is surely to be expected, but the unexpected conclusions are that the Bayesian approach (i) works well even in the zero-noise PMS and (ii) is, in the case studied here, able to distinguish the PMS from the IMS.

\section{ACKNOWLEDGMENTS}

This publication was based on work supported in part by Award No KUK-C1-013-04, made by King Abdullah University of Science and Technology (KAUST).

\section{REFERENCES}

1. L. M. Berliner, Journal of the American Statistical Association 416, 938-952 (1991).

2. K. Judd, and L. A. Smith, Physica D 151, 125-141 (2001).

3. K. Judd, and L. A. Smith, Physica D 196, 224-242 (2004).

4. C. Beck, and F. F. Schögl, Thermodynamics of Chaotic Systems: An Introduction, Cambridge, England: Cambridge University Press, 1993.

5. K. Judd, Phys. Rev. E 67 (2003).

6. D. Pollard, A User's Guide to Measure Theoretic Probability, Cambridge, England: Cambridge University Press, 2002. 



\section{RECENT REPORTS}

12/10 Nonlinear Morphoelastic Plates I: Genesis of Residual Stress

McMahon

Goriely

Tabor

13/10 Nonlinear Morphoelastic Plates II: Exodus to Buckled States

McMahon

Goriely

Tabor

14/10 Analysis of Brownian dynamics simulations of reversible biomolecular reactions

Lipkova

Zygalakis

Chapman

Erban

15/10 Travelling waves in hyperbolic chemotaxis equations

Xue

Hwang

Painter

Erban

16/10 The Physics and Mechanics of Biological Systems

Goriely

Moulton

17/10 Crust formation in drying colloidal suspensions

Style

Peppin

18/10 A Mathematical Model of Tumor-Immune Interactions

Robertson-Tessi

El-Kareh

Goriely

19/10 Elastic cavitation, tube hollowing, and differential growth in plants and biological tissues

Goriely

Moulton

Vandiver

20/10 Asymptotic expressions for the nearest and furthest dislocations in a pile-up against a grain boundary

21/10 Cardiac electromechanics: the effect of contraction model on the mathematical problem and accuracy of the numerical scheme

Pathmanathan

Chapman

Gavaghan

Whiteley

22/10 Fat vs. thin threading approach on GPUs: application to stochastic simulation of chemical reactions

Klingbeil

Erban

Giles

Maini

23/10 Asymptotic analysis of a system of algebraic equations arising in dislocation theory

Hall

Chapman

Ockendon

25/10 Preconditioning for Allen-Cahn Variational Inequalities with NonLocal Constraints

Blank

Sarbu

Stoll

26/10 On an evolution equation for sand dunes

Ellis

Fowler

Benilov

Chapman 
29/10 A Priori Error Estimates for Semidiscrete Finite Element Approx-

Goswami imations to Equations of Motion Arising in Oldroyd Fluids of Order One

Pani

30/10 The Landau-de Gennes theory of nematic liquid crystals: Uniaxiality versus Biaxiality

31/10 The Radial-Hedgehog Solution in Landau-de Gennes' theory Majumdar

32/10 Nonlinear instability in flagellar dynamics: a novel modulation Gadelha mechanism in sperm migration?

Gaffney

Smith

Kirkman-Brown

33/10 Error bounds on block GaussSeidel solutions of coupled multiphysics problem

Whiteley

Gillow

Tavener

Walter

34/10 A random projection method for sharp phase boundaries in lattice

Reis Boltzmann simulations

Dellar

35/10 Regularized Particle Filter with Langevin Resampling Step

Duan

Farmer

Moroz

Copies of these, and any other OCCAM reports can be obtained from:

Oxford Centre for Collaborative Applied Mathematics

Mathematical Institute

24 - 29 St Giles'

Oxford

OX1 3LB

England

www.maths.ox.ac.uk/occam 